\title{
Salvatore Veca per noi
}

\author{
di Emilio Renzi \\ emilio.renzi@gmail.com
}

\begin{abstract}
Salvatore Veca (31 October 1943 - 7 October 2021) was not only a lecturer in many universities, including eventually Pavia, but also an active intellectual in many important civic structures. A brilliant pupil of Enzo Paci at the University of Milan, editor of the journal «aut aut», Veca chaired the Fondazione Feltrinelli and the Casa della Cultura in Milan and was the author of many books on philosophy based on the critical introduction of Anglo-Saxon analytical culture. The presentation in Italy of the work of John Rawls was important and marked the liveliest interest in the problem of the "just society". He played a leading role in the debate on the possible developments of the Italian left after the fall of the Berlin Wall. For the Expo Laboratory he was responsible for drafting the "Milan Charter" on environmental sustainability.
\end{abstract}

Keywords: Enzo Paci, aut aut, Annali della Fondazione Feltrinelli, John Rawls, Carta di Milano sulla sostenibilità ambientale

La morte sigilla per i filosofi e per la cultura milanese e italiana tutta il bel sorriso gentile di Salvatore Veca. A maggior ragione apre ora la riflessione su un pensiero ricco di molti motivi, che spaziano dalla filosofia che è sempre ricerca che si rinnova ai problemi di una società giusta.

La filosofia di Salvatore Veca espressa in molto libri e saggi sta fondamentalmente sotto il segno della "utopia possibile". Nel tempo che stiamo vivendo, come persone e come istituzioni, nulla è dato una volta per sempre, al contrario la ricerca filosofica è possibilità che continuamente rinnova se stessa. Antidogmatismo e possibilità sono dunque le due parole chiave.

Vi è eleganza nel modo in Veca cui parlò di sé e dei suoi anni pubblici a vita piena nel suo ultimo lavoro, Prove di autoritratto, con Sebastiano Mondadori (Mimesis, Milano 2020). Perché "prove"? Vuol dire non parlare mai del mondo e di stessi in modo asseverativo, ideologico, o comunque egocentrico, ma 
sempre implicando le ragioni e le esistenze di ogni altro o altra con cui si abbia avuto a che fare.

La prima "prova", iniziatica e formativa, è la scelta di fare Filosofia all'Università statale di Milano. Filosofia è la fenomenologia husserliana insegnata da Enzo Paci, il migliore degli allievi di Antonio Banfi, che difatti Veca scelse ed ebbe maestro per i non pochi anni in cui la rivista «aut aut» fondata da Paci fu realizzata in collaborazione editoriale anche con Pier Aldo Rovatti. Paci lo incitò a un seminario di fenomenologia del teatro, che lo mise in un fertile contatto con Paolo Grassi e Giorgio Strehler. Era la Milano che si apriva e sviluppava lasciandosi alle spalle il dopoguerra.

È anche la fase dell'incontro con amici e sodali di idee, soprattutto Fabrizio Mondadori, il primogenito di Alberto Mondadori editore del Saggiatore.

Dopo l'opera capitale Funzione delle scienze e significato dell'uomo del 1964 Paci pose mano a una operazione filosofica e politica cercando la convergenza tra l'ultimo Husserl e il marxismo, soprattutto quello dello dei Manoscritti economico-filosofici di Marx. Al riguardo Veca scrive: "Il tentativo di contaminazione mi lasciò piuttosto perplesso... quando intorno al '72 Paci e Rovatti tendevano a imprimere alla rivista un indirizzo politico, di influenza marxista, che guardava alla sinistra extraparlamentare, nel pieno di una fase fortemente ideologizzata che contrassegnò i primi anni Settanta, retaggio del lungo Sessantotto italiano...io non condividevo la nuova linea. Il rapporto tra politica e cultura deve preservare la ricerca intellettuale preservando una relativa autonomia rispetto alla prassi politica. Siccome questa autonomia politica veniva a mancare nel nuovo orientamento di "aut aut", e mi trovavo in minoranza rispetto a Paci e a Rovatti, senza traumi posi fine al lungo sodalizio con un grande maestro e un grande amico».

Il primo libro di Veca nel 1967 e si intitola Fondazione e modalità in Kant. È significativa la scelta di un autore classico e al suo interno il tema della "modalità". Il tema della logica modale non sarà mai abbandonato. Il successivo Saggio sul programma scientifico di Marx desterà echi diversi per 
le implicazioni critiche che aveva per il marxismo, di cui era imbevuta certa filosofia e certa politica di allora.

Molto opportunamente Franco Sarcinelli, direttore della rivista online "InCircolo», ha ricordato un incontro di studiosi e meno giovani.

Il titolo dell'incontro era "Il senso della possibilità", come anticipazione di un libro che stava scrivendo per Feltrinelli, e che poi venne pubblicato con quel titolo nel 2018.

Le sue parole di esordio furono queste: «Cominciamo con il senso della possibilità, abbozzando qualcosa come dei frammenti di un discorso che chiamerò per convenzione utopico, in cui si delineano mondi sociali possibili», e citò l'incipit di una poesia di Emily Dickinson:

Io abito la Possibilità

Una casa più bella della prosa più ricca di finestre superbe le sue porte.

Continuò raccogliendo voci filosofiche come esclamate da altrettante finestre aperte sul mondo, preconizzando assetti sociali possibili da sperimentare nel futuro: Jon Elster in Making sense of Marx, Robert Nozick in Anarchia, stato e utopia, Max Weber in La politica come professione, e John Rawls in Il diritto dei popoli.

Il suo contributo è stato decisivo per aprire la filosofia italiana ai venti anglosassoni in un senso più ampio, in forma di traduzione di testi di autori che, se oggi sono noti al grande pubblico italiano, lo devono a lui: John Rawls, Ronald Dworkin, Michael Walzer, Isaiah Berlin, e molti altri, sono disponibili al lettore italiano anche grazie alla sua capacità di capire che cosa, in filosofia in generale e in filosofia politica in particolare, contasse davvero, chi - per usare una sua espressione - «ci acchiappasse». Questo per il filosofo, non poteva avvenire se non attraverso la chiarezza del pensiero e il rigore del lavoro argomentativo, che soli potevano produrre un solido apparato giustificativo a sostegno degli ideali politici. 
Questo naturalmente aveva una serie di conseguenze sull'impostazione filosofica generale, ma aveva anche delle ricadute nel dibattito politico italiano, fin quando almeno nei partiti si è dato ascolto agli intellettuali, eventualmente inferocendosi per quel che gli intellettuali facevano o dicevano, specialmente se scattava il sospetto di non conformità all'ortodossia ideologica. E la figura di Salvatore Veca è stata quella di un intellettuale nel senso pieno della parola; che ha cercato negli anni di far ragionare la politica.

Il posto di Salvatore Veca nel repertorio della filosofia contemporanea è stato fissato precocemente come quello del "neocontrattualista" che importava dagli Stati Uniti e adattava originalmente all'Europa la teoria della giustizia (una teoria della giustizia, mi avrebbe certamente corretto) di John Rawls del 1971. Visto ora a distanza, quello era in realtà il suo lavoro giovanile, di straordinaria importanza anche per gli effetti che avrebbe avuto nell'influire sul corso delle idee in Italia e in particolare sulla sinistra, ma un'opera a cui molto sarebbe seguito nella prolifica e sostanziosa produzione, con la lunga stagione dei successivi lavori sui temi dell'incertezza, dell'incompletezza, e con loro del pluralismo, che caratterizza il sapere e la condizione degli esseri umani. Si tratta della stagione della maturità piena del Veca filosofo della politica e della conoscenza che, a 77 anni, molto avrebbe avuto ancora da dire.

Non solo Rawls dunque. È tornata in questa stagione la ricchezza della sua formazione negli anni Sessanta all'Università statale di Milano, dove l'ho conosciuto, da matricola io, lui di pochi anni più vecchio, ma già per me un "professore" come assistente di Enzo Paci. Una formazione in cui ha contato quella forte voce teoretica che insegnava, in modo suggestivo e visionario la fenomenologia di Husserl, e che spingeva ad affrontare con rigore analitico le filosofie del linguaggio, Quine, la logica. Milano era allora un incredibile serbatoio di risorse intellettuali: con Paci, c'erano Ludovico Geymonat a filosofia della scienza, Mario Dal Pra a storia della filosofia, Ettore Casari alla logica e Cesare Musatti E Musatti fu anche per lui studente, come per tanti, il primo degli esami, la prima passione accademica. Salvatore lo racconta, 
nell'ultimo suo libro, l'autobiografia scritta nei mesi del ritiro da Covid, insieme al figlio di sua moglie Nicoletta Mondadori, Sebastiano (Prove di autoritratto). Forse anche per questo egli vedeva nel Sessantotto un momento di svolta significativo non per la politica ma per i mores, il mutamento di ordine sociale, la liberazione sessuale, l'innesco di una rivoluzione femminista.

È stato costante nella vita di Veca l'impegno pubblico, nei confronti della politica, rispetto alla quale ha sempre difeso l'autonomia del pensare, in questo seguendo la lezione di Norberto Bobbio: impegno ma con distanza. Una distanza che non gli ha risparmiato polemiche anche molto dure da parte dei settori più conservatori del Pci. Quando presentò all'Istituto Gramsci di Bologna, nel 1982, il suo lavoro sulla società giusta, che tradotto in politica significava il riformismo per una società "migliore", fu letteralmente assalito come "traditore" dei principi rivoluzionari e della classe operaia. Ma questo non impedì al suo pensiero di attecchire e sulla distanza di conquistare consensi sempre più larghi e alla fine prevalenti. Fu anche con il suo aiuto personale che, nel mio lavoro all'Unità, potei prima far circolare i nomi e le idee, allora una novità non indolore nel contesto dei comunisti italiani, della cultura liberale di Walzer, Rawls, Amartya Sen e tanti altri. Si consolidava con la sua spinta personale, certo insieme a Bobbio, a Vittorio Foa e Giuliano Amato, con Michele Salvati, Alberto Martinelli, Giovanna Zincone, Guido Martinotti, Nadia Urbinati, Marina Calloni e Elisabetta Galeotti una larga corrente di pensiero di ispirazione liberalsocialista, che avrebbe dato vita con chi vi scrive alla rivista «Reset».

Non erano mancate le tensioni nei confronti del Pci fin da quando Salvatore, insieme a Salvati, ne aveva chiesto l'abbandono del nome. Ma le resistenze servirono soltanto a ritardare quel che comunque avrebbe dovuto avvenire. "Tentavamo di fare da ponte tra l'Avanti! e l'Unità», racconta nell'autobiografia, "Ardua impresa negli anni Ottanta!». E a proposito di abbagli circa il Veca "traditore" è da rimarcare che le sue prese di posizione 
più recenti avrebbero stupito se mai per il loro ancoraggio a una visione radicata circa la funzione della sinistra, del sindacato, della giustizia sociale.

È da ricordare il lascito enorme di Veca accademico, insegnante, guida dell'istituto superiore di Pavia, e poi anche editore con la casa editrice Feltrinelli, e organizzatore di cultura alla testa della Fondazione Feltrinelli, dei suoi Annali e dei suoi incontri. Ma dovremo a lungo rileggere i suoi lavori del ciclo dell'incertezza e dell'incompletezza.

Insieme a molteplici influenze ritorna nelle pagine degli ultimi vent'anni con grande forza quella di Isaiah Berlin, non solo e non tanto il Berlin dei saggi sulla libertà (negativa), ma soprattutto quello del pluralismo dei valori: "Altre vallate altre pecore", diceva con una battuta alludendo alle varietà delle culture umane, ed echeggiando Pascal e Montaigne.

Consapevole della impossibilità di stringere nelle nostre mani una compiuta ed esauriente conoscenza della verità, il filosofo aveva aperto una nuova fase della sua ricerca che voleva integrare la sua idea della giustizia con una prospettiva di realismo filosofico. Una visione della filosofia come permanente "lavoro in corso", aperta sempre e dovunque si percepisca l'esistenza di un problema, sempre alle prese con la difficoltà del fondamento, come i marinai sulla "barca di Neurath" (Otto, il filosofo austriaco, ideatore dell'immagine), «sempre alle prese con uno scafo da rimodellare senza mai poterlo mettere in cantiere per ricostruirlo con materiali migliori». Opera da continuare per "coltivatori di memorie" (la storia e la storia delle idee) ed "esploratori di connessioni", le idee che ci servono per andare avanti.

Presidente onorario della Fondazione Feltrinelli, al timone della Casa della Cultura e del Premio Balzan, alla sua città ha donato "La Carta di Milano" durante l'Expo. "Amico carissimo e pensatore originale», lo piange anche il presidente emerito Giorgio Napolitano, ricordando la loro vicinanza «in una visione riformista della sinistra italiana e nello sforzo di rinnovarla».

L'ultima fatica sono state queste parole, scolpite come versi da far volare nel cielo di Milano: «portale alla Shammah», mi ha detto, «e leggile per me, il 
teatro è il luogo della cultura e dell'incontro, immaginatemi in sala, felice, ad ascoltare». Al Parenti le sue parole messe in fila hanno commosso e toccato il cuore, sono diventate inno, manifesto, anzi un canto per la cultura, quando hanno evocato la metafora cara a Carlo Cattaneo e ripresa da Norberto Bobbio, l'orchestra dei passerotti da contrapporre all'altezzosità rapace delle aquile: quando si parla di cultura vengon fuori le aquile, diceva Cattaneo, ma io vorrei dei passerotti... Anche oggi, ha rilanciato Salvatore Veca, alla vanagloria delle aquile bisogna contrapporre l'orgoglio dei passerotti, alla cultura accademica si deve preferire quella che nasce dal basso, dalle botteghe, dai quartieri, dagli ospedali, dai luoghi marginali, dalla fatica, dalla vita vissuta, dalle città... È sembrato un auspicio profetico, un invito a riconnettere le diverse Milano spezzate dalle diseguaglianze e dalle sofferenze del Covid, ma era anche un messaggio in bottiglia alla «Milano altra», come la chiamava lui, e agli amici riuniti per una serata dedicata alla cultura, inventata dalla passione civica di Andrée Ruth Shammah. È stato l'ultimo esercizio di immaginazione urbana di un grande filosofo, generoso, profondo, geniale nelle intuizioni, maestro amato e riconosciuto da generazioni di studenti, impegnato sul fronte dell'economia civile e della buona politica, quella che negli ultimi tempi incoraggiava a indossare i colori della ripartenza: il verde dell'ambiente e il blu del digitale, necessari per stemperare i colori del vecchio mondo e creare un «daltonismo globalizzante», che per Veca significa capitalismo paziente e non predatorio, lotta alle diseguaglianze, connessione tra salute e ambiente urbano.

Ecco le parole da salvare, così come le ha dettate, scritte per Milano ma destinate all'Italia e a chi cerca vie nuove di rinascita, come lui ha sempre fatto con le lezioni universitarie, i dibattiti alla Casa della Cultura, i libri, gli incontri alla Fondazione Feltrinelli, gli articoli sui giornali. Al «Corriere», ricordava, venne chiamato per ringiovanire con le nuove idee la cultura, insieme alla pattuglia di intellettuali che nei primi anni Settanta rivoluzionò la Terza pagina: Eco, Calvino, Arbasino, Pasolini, Fortini, Ginzburg, Musatti... Erano 
suoi i temi della società giusta, del rapporto tra equità e libertà e tra filosofia e politica, che per Veca doveva avere un solo aggettivo: buona. «Le parole volano», mi ha spiegato con un sorriso mente le dettava, seduto sul divano di casa, a fianco della moglie Nicoletta che vigilava con dolcezza sui tempi strappati alle cure. «Era una sera di fine settembre, all'ora del tramonto, e saremmo andati avanti per ore a discutere del cambiamento che riteneva necessario dopo il trauma della pandemia: non per cancellare la storia, ma per scriverne un'altra, per fare memoria del passato ed entrare nel portale del nuovo mondo da uomini e donne liberi e coscienti, senza essere prigionieri né di Internet, né di Facebook, né dei pregiudizi. Mi raccomando», ha concluso, «questo è solo un inizio: la parola "continua" va scritta dai giovani».

\section{La gentilezza}

Serve una trasmissione gentile dei saperi: gentilezza vuol dire basta odio, basta no. La dobbiamo verso il nuovo, le idee, le forme, le pratiche, e le parole dismesse dei vecchi vocabolari, le abitudini abbandonate, le credenze e i valori, su cui abbiamo imparato a sospendere - esitanti, severi o critici - il giudizio abituale per impratichirci del nuovo.

\section{Il rispetto}

Verso il nuovo e il suo corteo luminoso e incerto, ancora una volta, ma con tutta la consapevolezza della posta in gioco, la fiducia, la scommessa e la promessa. Ci serve un nuovo vocabolario del nost Milan.

\section{Le scienze}

La vocazione, le regioni scientifiche della cultura ambrosiana, il suo esprit de geometrie non sono certo cose inedite, ma inaspettato è l'effetto mosaico con le tessere nuove della città che si reinventa. 


\section{Le arti}

Lo stesso pensiero ci accompagna quando ci immergiamo nello spazio mutevole dei linguaggi dell'arte. Scienze e arti sono il gesto del ricorrente e sempre nuovo omaggio a Leonardo.

\section{Le tecnologie}

Sappiamo che può accadere che le tecnologie vadano strettamente in tandem con le scienze e le arti e che, in quel caso, i confini siano porosi e vi sia metamorfosi. Sosteniamo che questo è e deve essere un must nella cultura ambrosiana.

\section{I saperi}

Saperi pratici, saperi teorici, saperi proposizionali, saper fare, congiurano luminosamente nel tessere le fila dell'arte della convivenza, che si realizza nelle regioni della saggezza, della giustezza, della bellezza a opera dell'orchestra dei passerotti di Carlo Cattaneo.

\section{La lealtà}

La lealtà è bifronte. Essa ci è nota come virtù che preserva tratti salienti del passato. Dobbiamo imparare, per prove ed errori, a connetterci con essa ai varchi del futuro. Al principio speranza.

\section{L'anima}

Noi crediamo che il corpo sia l'immagine perspicua dell'anima e, ciascuno a modo nostro, sentiamo o dobbiamo tornare a sentire che lo spirito soffia dove vuole. L’anima di Milano non sai dove cercarla, ma sai che ti può capitare di trovarla. E quella rappresenta davvero il nost Milan. 


\section{Il corpo}

Pensando all'anima, pensiamo al corpo e questo lo rende luminoso. Così, abbandonando esangui e arcaici pregiudizi, avvertiamo la connessione vivente fra etica e estetica. A Milano vien da pensare alla moda, alla creatività e al suo essere anche cultura. Il Covid ci ha ricordato quanto è miope e filosoficamente ottuso sottovalutare il nostro essere, in quanto esseri umani in carne e ossa, esposti alla sorte e all'imprevisto. Noi non abbiamo corpi, noi siamo corpi. Allo stesso modo noi non abitiamo la Terra, noi le apparteniamo.

\section{L'umanità}

La ragione pubblica e civile rinvia dal locale al globale e ci ricorda una massima alla quale attenersi: una sola umanità, un solo pianeta. Oggi siamo chiamati a costruire la costellazione della solidarietà, scavalcando gli operatori della lacerazione e della scissione dello spazio d'umanità, minato da razzismo, sessismo, classismo, nazionalismo, fondamentalismo. In molti luoghi del pianeta bisogna chiedersi con Primo Levi «se questo è un uomo»: guerre, massacri, deportazioni, sfruttamento, barbarie, torture e schiavitù. Io credo nella democrazia accessibile e negli spazi pubblici della «Milano altra», dove si è differenti e uguali, dove tutti possono sentirsi della stessa età, anche chi è più vecchio o più giovane degli altri. Non voglio pensare al destino cinico e baro, ma alla cultura che accoglie e non accetta le ineguaglianze. 